\title{
Changes of pelvis control with subacute stroke: A comparison of body-weight- support treadmill training coupled virtual reality system and over-ground training
}

\author{
Yurong Mao, Peiming Chen, Lifang Li, Le Li* and Dongfeng Huang* \\ Department of Rehabilitation Medicine, First Affiliated Hospital, Sun Yat-Sen University and \\ Guangdong Provincial Research Center for Rehabilitation Medicine and Translational Technology, \\ Guangzhou, Guangdong, China
}

\begin{abstract}
.
BACKGROUND: Gait recovery is very important to stroke survivors to regain their independence in activity of daily life. OBJECTIVE: This study aimed to investigate the effects of virtual reality (VR) coupled body weight support treadmill training (BWSTT) on pelvic control at the early stage of stroke.

METHODS: Kinematic and kinetic changes of pelvic motion were evaluated by a 3D gait analysis system and were compared to the results from over-ground walking training. Twenty-four patients having unilateral hemiplegia with subacute stroke were recruited to a VR coupled BWSTT group $(n=12)$ and a conventional therapy (CT) group $(n=12)$. Both of the groups received training of $20-40 \mathrm{~min} /$ day, 5 days/week, for 3 weeks.

RESULTS: The results showed the tilt of pelvis in sagittal plane improved significantly $(P=0.038)$ after treatment in the BWSTT + VR group, in terms of decreased amplitude of anterior peak (mean, from $10.99^{\circ}$ to $6.25^{\circ}$ ), while there were no significant differences in the control group.

CONCLUSION: The findings suggested that VR coupled BWSTT gait training could decrease anterior tilt of pelvis in early hemiparetic persons following a modest intervention dose, and the training may have advantages over conventional over-ground gait training and can assist the therapists in correcting abnormal gait pattern of stroke survivors.
\end{abstract}

Keywords: Body weight support, gait analysis, stroke, pelvic control, virtual reality

\section{Introduction}

Pelvic motion, which contains two units of passenger and locomotor, is very important in gait economy [1]. Patients with stroke usually show a hemiplegic gait with the asymmetrical pattern. The primary gait asymmetry can lead to the development of compensatory motor behaviors, and the gait deviations such as knee flexion decreasing during stance phase often bring some negative effects on the control of

\footnotetext{
${ }^{*}$ Corresponding author: Le Li and Dongfeng Huang, Department of Rehabilitation Medicine, First Affiliated Hospital, Sun Yat-Sen University and Guangdong Provincial Research Center for Rehabilitation Medicine and Translational Technology, Guangzhou, Guangdong 510080, China. Tel.: +86 02087332200 8536; Fax: +86 020 87750632; E-mail: lile5@ mail.sysu.edu.cn, huangdf@mail.sysu.edu.cn.
} 
pelvis [2]. Pelvic elevation and tilt angle increasing are described with stroke hemiplegic gait, and the poor motor function with stroke patients lead to excessive pelvis tilt during the stance and swing phase. These changes reduce the stability [3-5]. In addition, the circumduction gait includes a combination of hiking and forward rotation of the pelvis [6]. These evidences highlighted that the sway amplitude and stability of pelvis are important in paretic patients, and could have an influence on circumduction gait pattern of lower limb and locomotor performance, as well as functional ability of upper limb activity and trunk control of stroke patients [7-9]. These studies emphasized the impact of pelvis on motor function and gait. However, there is still a lack of evidence based on quantitative three dimensional analysis of pelvic motion changes, especially in early stage of stroke.

In order to facilitate locomotion and improve walking ability, physical therapy intervention in restoring motor function is widely considered to be beneficial in the treatment of patients with stroke in early stage [10[11], and the clinical outcomes of rehabilitation techniques have also been demonstrated [12[13]. However, effect of improving movement with stroke survivors are controversial [14 [15], and many results of clinical measurement with varying kinds therapy practice lacked quantitative difference. There are few studies exploring the pelvic motion changes in early stage of stroke after rehabilitation intervention.

Virtual reality (VR) has been used for the rehabilitation of neurological patients. Some studies have demonstrated the effect on postural control and motor functional improvement [16/17]. Taken as an interesting and motivating training practice with innovation technology, VR facilitated the improvement of walking ability [18]. Body-weight-support treadmill training (BWSTT) is a task-specific and repetitive nature of step training on the treadmill with partial body weight of the subjects been held [19]. It enables the harness-secured patients to practice numerous steps assisted by therapists at an early stage after neurological insult, and the assisted control by therapist focuses on the pelvis of proximal limbs [20]. Literature results showed BWSTT improved walking speed, motor function and balance ability on stroke patients [2122]. Moreover, VR coupled BWSTT had demonstrated its feasibility, and the results of clinical scales showed the improvement of the gait and balance for persons after stroke [23|24]. In this study, a VR environment system on the TV screen before treadmill combined BWSTT was applied to assist therapists in training subacute stroke patients with gait deficits and the results were analyzed by quantitative motion analysis.

In literature, the impact of treadmill and ground walking on the pelvic girdle mobility with healthy subjects, was investigated by a synchronous three-dimensioned motion analysis, and the results showed an active increase in the sagittal plane and a passive increase in transverse plane in the pelvis range of motion (ROM), as well as a significant anterior tilt increasing of pelvis [1]. Compared to over-ground training, there were the detected kinematic difference and the pelvic tilt increasing on treadmill training. Therefore, it is necessary to conduct the research of the impact of treadmill training on pelvic girdle on stroke patients in early stage. Three-dimensional gait analysis is the gold standard for evaluation of gait abnormalities [25], and is often used to quantitatively describe the gait and joint angle changes in hemiplegic patients [26]. Therefore, the objectives of this study were to observe and compare changes in pelvis in three planes (sagittal, frontal, horizontal) during walking at self-selected speed after the intervention of VR coupled BWSTT and to study the influence on hemiplegic gait. The hypothesis of this study is that by using the standard three-dimension kinematic analysis, the changes of pelvic girdle could be determined, and the findings could be used to evaluate whether the pelvic anterior tilt increasing or not after VR combined with BWSTT on person after stroke in early stage. This outcome may also help to assess clinical effects and explain the biomechanical mechanism of BWSTT on improving gait pattern of persons after stroke. 
Table 1

Demographic and clinical features of participants

\begin{tabular}{llll}
\hline Demographics and eligibility & VR + BWST $X(\mathrm{SD})$ & CT $X(\mathrm{SD})$ & $P$ \\
\hline Age, $y$ & $58.18(11.15)$ & $63.09(11.51)$ & 0.378 \\
Days of post-injury & $48.91(17.01)$ & $48.91(17.92)$ & 1.00 \\
Leg affected, no (\%) of left & $6(55 \%)$ & $6(50 \%)$ & \\
Cerebral infraction, no (\%) hemorrhage & $10(91.7 \%)$ & $10(83.3 \%)$ & \\
MMSE score, 0-30 & $28.45(1.37)$ & $28.36(1.21)$ & 0.887 \\
Body weight (kg) & $66.91(10.23)$ & $65.27(11.97)$ & 0.799 \\
Body height (mm) & $1664.6(73.94)$ & $16366.8(101.35)$ & 0.482 \\
Leg length (mm) & $837.3(62.26)$ & $823.2(57.63)$ & 0.559 \\
\hline
\end{tabular}

\section{Material and methods}

\subsection{Participants}

Patients suffering subacute stroke were recruited from the inpatient department of Rehabilitation Medicine at the First Affiliated Hospital, Sun Yat-sen University. Twenty-four patients were recruited according to the following criteria: (1) stroke confirmed by CT or MRI, stable vital sign, age between 40 years-78 years; can walk independently with 10 meters. (2) unilateral hemiparesis for no more than 3 months resulting from first stroke insult; (3) residual gait impairment, decreased walking speed (age < $60=10$ seconds or longer or $1 \mathrm{~m} / \mathrm{s}$, age 60 to $69: 12.5$ seconds or longer or $0.8 \mathrm{~m} / \mathrm{s}$, age $\geqslant 70: 16.6 \mathrm{sec}-$ onds or longer, $<0.6 \mathrm{~m} / \mathrm{s}$ ), (4) adequate mental and physical capacity to attempt the tasks as instructed (MMSE $\geqslant 27$, MAS $\leqslant 2$ ). Patients were excluded from the study if they had: (1) A history of recent deep vein thrombosis of the lower limbs; (2) Combining other neurologic or orthopedic pathology; (3) Serious amblyopia, macula flava, retinopathy and defect of visual field. This study was approved by the Human Subjects Ethics Subcommittee of the First Affiliated Hospital, Sun Yat-Sen University, and written informed consent was obtained from each patient. Table 1 shows the clinical characteristics for the patients after stroke.

\subsection{Experimental design}

VR coupled BWSTT and three-dimension kinematic analysis of pelvic girdle were accomplished in Motor Recovery Research Laboratory of the hospital. A single blinded randomized control study design was employed. The flow chart describing the participation in the various stage of the study was shown in Fig. 1. Patients were divided into BWSTT coupled VR (VR + BWSTT) group ( $n=11,9$ male, 2 female, 41 age -72 age) and conventional therapy (CT) group ( $n=12,9$ male, 3 female, 45 age-76 age). Patients were evaluated before intervention and after three-week training program.

A motion analysis system (Vicon MX13, VICON Peak, Oxford, UK) with six infrared $100 \mathrm{~Hz}$ cameras was used to record the angle of pelvic movement. The data were the locations of 16 reflective markers taped to the skin overlying bony landmarks of the pelvis and lower limbs [27], including the midline sacrum at the level of the posterior superior iliac spines, anterior superior iliac spines, lower lateral 1/3 and $1 / 2$ surface of left and right thigh, lateral epicondyle of knee, lower lateral $1 / 3$ and $1 / 2$ surface of left and right shank, lateral malleolus, the second metatarsal head, and the calcaneus at the same height as the toe marker. The captured data were subsequently analyzed using Vicon Nexus (Version 1.7.1) and Plug-in-Gait model (Fig. 2). 




Fig. 1. Flow chart describing participation in the various stage of the study.
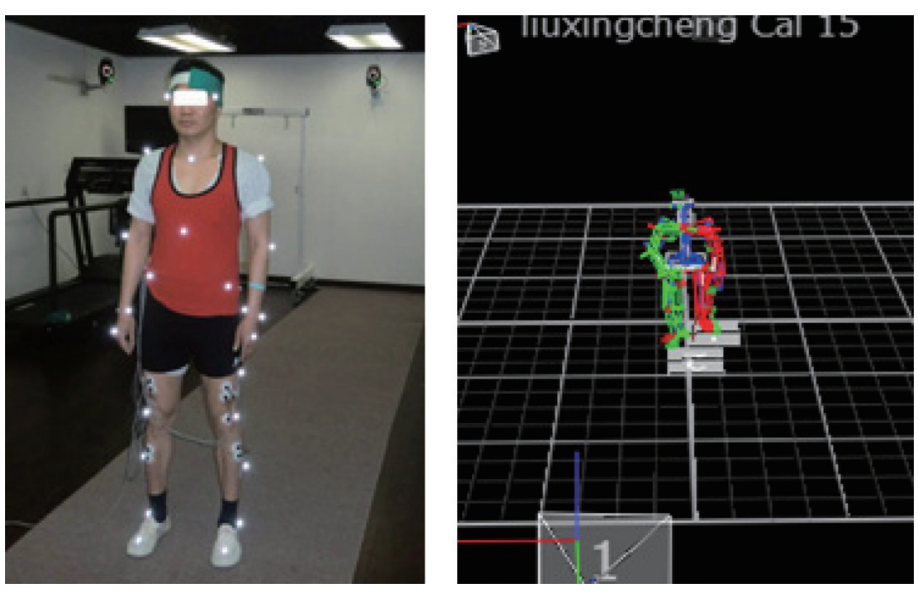

Fig. 2. Kinematic capture using vicon and plug-in gait.

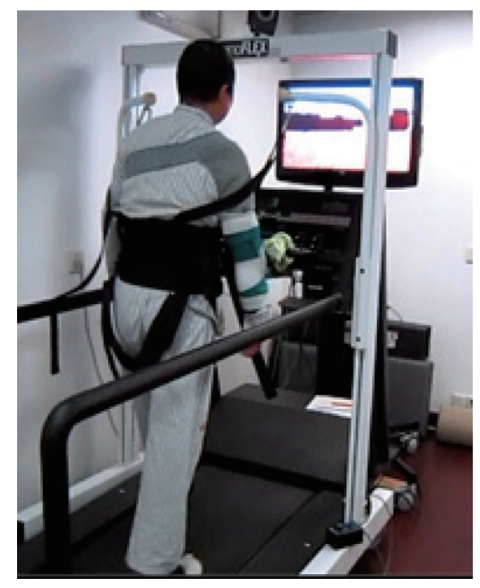

Fig. 3. VR + BWSTT training.

All subjects walked without canes, orthosis or any other assisted devices. During the tests, the patients were asked to walk back and forth at a self-selected walking speed and habitual posture on a $10 \mathrm{~m}$ walkway. Five successful walking trials defined as consistent trajectories were collected at baseline condition and after 3-week training. Marker trajectories were sampled at $100 \mathrm{~Hz}$ for the calculation of lower extremity joints angle. Each walking trail was normalized and represented in percentage over the course of the gait cycle, and a total of 51 points of data of a full gait cycle from foot strike to next foot strike with $2 \%$ gait cycle increment were calculated. The pelvic girdle mobility of three planes (sagittal, frontal and horizontal) were recorded [28].

\subsection{Intervention}

The treatment group received walking training with BWSTT coupled VR for 30 minutes, 2-3 ses- 
sions every day, five days a week for three weeks (Fig. 3). The equipment for BWSTT was from a standard treadmill (NoramcoFitness Inc, TX) combined to weight supporting apparatus (SpinoFlexcInc, NH, USA). The VR hardware components included a 40-inch television installed on a standing frame in front of the treadmill to display the VR environment, and the television was connected to a computer and the treadmill. The VR programs (visual trainer) consisted from a series of video shows (climbing mountain, crossing street, park, stadium, etc.), and the speed of video shown on screen was synchronized with treadmill velocity (Fig. 3). The initial body weight support amount was set at 30\% 40\%, and the speed of the treadmill was set at $0.5 \mathrm{mph}$ (miles per hour) [29]. The training intensity at first week was around $10 \mathrm{~min}-20 \mathrm{~min}$, then increased at $30 \mathrm{~min}$ in the third week, while the treadmill speed increased to $2.0 \mathrm{mph}$. The patient wore a modified mountain climber's harness with an adjustable belt around the pelvis and thigh, and an adjustable belt above to support the body weight. The physiotherapist assisted to control the lower extremity if the patient could not lift paretic leg. At the beginning of training, some patients need two therapists to guide the movement of pelvis forward and the hemiplegic leg flexion during the swing phase and extension at the stance phase. The BWS gradually decreased and the velocity also gradually increased in order to attain the maximum of the patient's walking ability, but the two parameters were not changed simultaneously. Heart rate and blood pressure were monitored in both groups before and after each session, using a digital sphygmomanometer, and also measured in pauses for taking a break with BWSTT.

The conventional group received individually walking training on the ground according to the Neurodevelopment therapy (NDT) instead of BWSTT for 30 minutes for 5 days, three weeks. Both groups received the same clinical treatment and the same amount of other therapy (e.g. physical agents, self exercises, occupational therapy), and scheduled 2-hour therapy program was allocated for each patient of physical therapy and implemented by physical therapist trained in the research protocol. All physical therapists documented participants' daily compliance with the study protocol. In addition, the whole rehabilitation team was educated concerning the experimental study protocol to ensure compliance when participants were not working with therapy staff.

\subsection{Statistical analysis}

Demographic date, including age, body weight, leg length and peak to peak of pelvis were evaluated and presented as mean \pm standard deviation. The three plane of pelvic trajectory was performed to compare the means. Independent $t$ test was applied on post hoc pairwise comparisons between different groups and paired T-test was applied on time effects comparisons with SPSS for Windows version 13.0. A $P$ value $<0.05$ was defined as a statistically significant difference for all statistical analyses.

\section{Results}

The mean values and the chart of the sagittal, frontal and horizontal pelvic motion curves of two groups before and after intervention are shown in Fig. 4. The peak-peak of two groups are compared in pre and post-training. Pelvic tilt in sagittal plane improved significantly $(P=0.038)$ after treatment, in terms of decreased amplitude of anterior peak (mean, from $10.99^{\circ}$ to $6.25^{\circ}$ ) in the BWSTT + VR group, but there was no significant difference at pelvic obliquity and rotation. For the CT group, there were no significant difference of pelvic peak changes before and after therapy. No significant difference of pelvic peak was found between BWSTT + VR and CT group after training (Table 2 and Fig. 4). 
Table 2

Comparison of pelvis kinetic high peak between pre and post-training of stroke patients

\begin{tabular}{llrcc}
\hline & & Pre-training & Post-training & $P$ \\
\hline VR + BWSTT & Tilt & $10.99 \pm 3.92$ & $6.25 \pm 5.90^{*}$ & 0.038 \\
& Obliquity & $6.05 \pm 7.01$ & $5.71 \pm 4.61$ & 0.894 \\
& Rotation & $5.79 \pm 5.89$ & $5.65 \pm 5.60$ & 0.957 \\
CT & Tilt & $7.13 \pm 3.17$ & $7.99 \pm 5.53$ & 0.655 \\
& Obliquity & $3.66 \pm 3.25$ & $2.71 \pm 5.82$ & 0.478 \\
& Rotation & $2.78 \pm 5.82$ & $3.97 \pm 4.25$ & 0.597 \\
\hline
\end{tabular}

*Significant difference $(P<0.05)$.
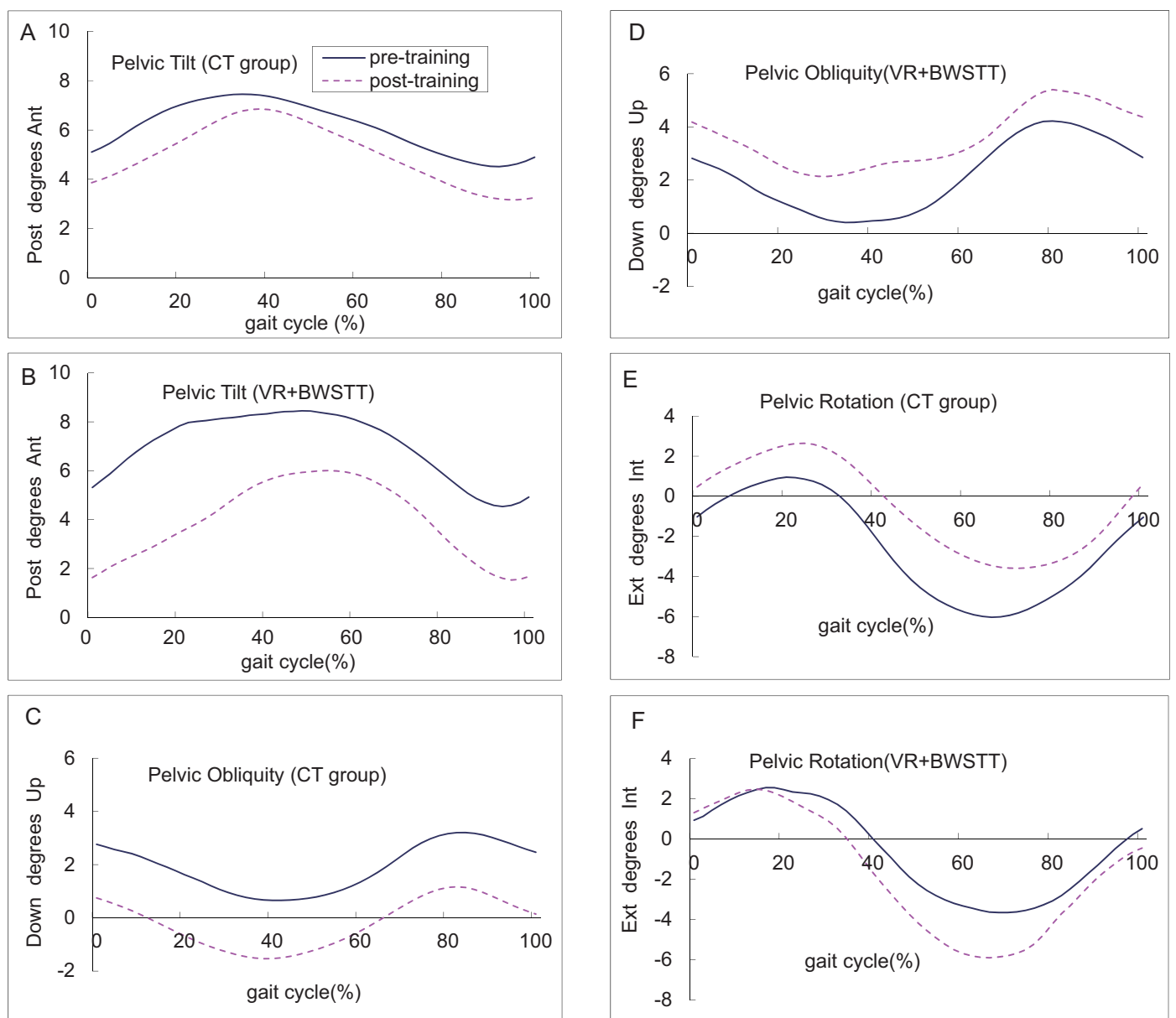

Fig. 4. Pre- and post-training with curves of pelvic angle with VR + BWSTT and CT group at the three plane (sagittal, frontal and horizontal).

\section{Discussion}

This study detected the difference in pelvic girdle mobility between VR coupled BWSTT and overground training and explored the feasibility in using VR coupled BWSTT to help prescribing and inducing near normal gait patterns to correct hemiplegic circumduction gait at early stage of stroke, especially 
adjusting the pelvic girdle motion. The differences of the pelvic girdle in VR coupled BWSTT and traditional training conditions were evaluated and compared.

As expected, this study found a significant decrease in pelvic anterior tilt peak angle after VR + BWSTT, and the curve trajectories showed the drop during the gait cycle, especially during stance phase (Fig. 4). However, this trend was not shown in CT group. Our findings of decreased the anterior tilt in pelvis after VR coupled BWSTT for three weeks supported the positive effects of the training., Dickstein and Abulaffio found both the anterior-posterior and mediolateral axes deviations of pelvis of hemiparetic patients had larger sways than normal subjects, and the sway in paretic side was larger than in the unaffected side [4]. Titianova and co-workers also described the pelvic elevation of the affected side during swing with hemiplegic patients, and demonstrated the correlation between the circumduction gait and pelvic deviation amplitude [5]. The decrease of the anterior tilt in pelvis would help the patient regain their normal gait pattern.

Previous study investigated the impact of surface on the changes of pelvic mobility in three planes of movement on the treadmill compared to walking on the natural ground in healthy subjects and 3D analysis only demonstrated a significant decrease on range of motion of pelvic girdle in the transverse plane on the treadmill, but there were no differences in the sagittal and frontal plane [1]. This might be related to the need of an active anterior tilt increase of pelvic girdle to adjust the walking on the treadmill with belt backward movement than on over-ground during gait cycle [1]. In contrast, the present results of this study demonstrated that there were significant differences in the sagittal plane pelvic girdle motion in treadmill group of subacute stroke survivors, and the curve showed anterior degrees decreased during the gait cycle. The anterior tilt of pelvic peak angle decreased and the curve trajectory dropped during the gait cycle might be caused by the treadmill belt transmitting rearward and the slower treadmill belt velocity. The pelvis and hip joint decreased forward flexion, as well as the hemiparetic lower extremity stepping forward for shorter step length need lesser forward flexion degrees [30]. Consequently, the results showed the anterior tilt trajectories dropped during gait cycle, especially a significant decrease during stance phase.

On the other hand, with the partial body weight supported, the hemiplegic limb could stepped forward easily and the frame of supported system controlled the trunk and pelvis forward lean. Hesse and coworkers observed more upright walking postures in hemiplegic subjects with body weight support [31], the stroke survivors decreased excessive flexion in pelvis and hip joint. In addition, the TV screen fixed with the frame in front of the treadmill in our study allowed the patients follow the scene in keeping straight of the upper part of body, and the VR training enhanced goal-oriented tasks rather than simple repetitive treadmill exercises for stroke survivors [23]. Compared to the healthy subjects, the hemiplegic patients showed increased pelvic tilt angle and circumduction gait [3]. The increased pelvic tilt induced knee flexion and made a contribution on decreasing the swing phase [6]. When the pelvis decreased the excessive anterior tilt, it should be the knee flexion increasing during the swing phase. Therefore, an improving gait with hemiparetic lower extremity appeared. However, subacute stroke patients with over-ground gait training in this study showed no significant difference in pelvic motion of sagittal plane. Previous studies also demonstrated BWSTT was superior to traditional gait training in improving motor function [23|30|32]. The above-mentioned reasons probably explained the targeted effect using BWSTT on gait parameters. Therefore, VR coupled BWSTT may be beneficial to correct abnormal circumduction gait pattern on the sagittal plane.

Robotic-assisted treadmill walking (such as Locomat ${ }^{\circledR}$ ) provides constant robot gravity compensation, and the inertia causes an increasing of ground reaction forces (GRF) to control pelvis moving up and down, and then is often applied in gait rehabilitation in stroke survivors [33]. Lokomat is usually 
used to focus on a symmetrical pattern [34] and previous studies mainly investigated the spatiotemporal parameters, as well as kinematic and kinetic parameters of hip, knee and ankle joint [35|36]. Finding of our study is comparable to the results from Locomat and may also contribute to explain the mechanism behind the robotic-assisted treadmill walking. For example, by testing healthy subjects on Locomat and analyzing the results from 3D kinematic and kinetic data, Swinnen and coworkers found a smaller antero-posterior and lateral translations of the pelvis and the trunk compare to traditional treadmill walking [37]. In line with this finding, our results also revealed a decreased sagittal plan of pelvic motion after the BWSTT, which supports the clinical usage of body weight support at early stage of stroke.

The data from our study indicated that there was no significant difference between the pelvic motion of frontal and horizontal of VR + BWSTT and in CT group (Table 2). In this study, patients can take self-selected comfortable walking speed at pre- and post-training, and the 3D kinematic analysis showed all patient' walking speed was lower $(<0.9 \mathrm{~m} / \mathrm{s})$ at subacute stage. The lower walking speed might cause compensation of thoracic motion to pelvic movement. In literature, it was showed that persons after stroke used more thoracic ROM than pelvic transverse ROM in comfortable and equivalent walking speed $(0.97 \mathrm{~m} / \mathrm{s}-0.98 \mathrm{~m} / \mathrm{s})$ compared to subjects without disability [38]. This may be the reason why we could not find any statistically significant differences in the peak angle of horizontal pelvic motion. Huang and the co-workers found that larger stride length and walking speed on the treadmill could produce large spinal and thorax-pelvic rotation, while small steps and speeding-up hardly affected thorax-pelvic-leg relative phase amplitudes, and stroke patients only showed thorax rotation to pelvis [39]. In line with their results, patients with stroke at early stage in this study usually started lower treadmill speed $(0.5 \mathrm{mph})$ and small step, which might contribute to the results that there was no significant increasing pelvic rotation.

This pilot study explored the pelvic peak and curve after VR + BWSTT and the over-ground walking intervention only with kinematic and kinetic data. Further studies combined with electrophysiology information (i.e. EMG) and muscles strength are necessary to help better understanding the mechanism about the control of pelvis with VR + BWSTT [38|39]. In addition, a larger sample size of random control trial study or multi-center design which compares VR + BWSTT, BWSTT only and over-ground training is warrant to draw further conclusion about the outcome of VR + BWSTT for gait disorder with persons after stroke.

In summary, the results in this study suggested that VR coupled BWSTT gait training could decrease anterior tilt of pelvis, and might have advantages over conventional over-ground gait training following a modest intervention dose and could assist therapists in correcting the abnormal gait pattern of the early hemiparetic patients.

\section{Acknowledgement}

This study is supported by the National Natural Science Foundation of China (No. 30973165, 31100669, and 81372108) and in part by Science and Technology Planning Project of Guangdong Province, China (No. 2013b090500099).

\section{References}

[1] Staszkiewicz R, Chwała W, Forczek W, Laska J et al. Three-dimensional analysis of the pelvic and hip mobility during gait on a treadmill and on the ground. Acta Bioeng Biomech. 2012; 14(2): 83-89. 
[2] Olney SJ, Griffin MP, McBride ID, Multivariate examination of data from gait analysis of persons with stroke. Phys Ther. 1998; 78(8): 814-828.

[3] Chen CL, Chen HC, Tang SF, Wu CY, Cheng PT, Hong WH, Gait performance with compensatory adaptations in stroke patients with different degrees of motor recovery. Am J Phys Med Rehabil. 2003; 82(12): 925-935.

[4] Dickstein R, Abulaffio N, Postural sway of the affected and nonaffected pelvis and leg in stance of hemiparetic patients. Arch Phys Med Rehabil. 2000; 81(3): 364-367.

[5] Titianova EB, Tarkka IM, Asymmetry in walking performance and postural sway in patients with chronic unilateral cerebral infarction. J Rehabil Res Dev. 1995; 32: 236-244.

[6] Perry J, Davids JR, Thorofare, NJ, Slac K, Gait analysis: Normal and pathological function. Journal of Pediatric Orthopaedics. 1992; 12(6): 815.

[7] Kerrigan DC, Frates EP, Rogan S, Riley PO, Hip hiking and circumduction: Quantitative definitions. Am J Phys Med Rehabil. 2000; 79(3): 247-252.

[8] De Bujanda ED, Nadeau S, Bourbonnais D, Dickstein R, Associations between lower limb impairments, locomotor capacities and kinematic variables in the frontal plane during walking in adults with chronic stroke. J Rehabil Med. 2003; 35(6): 259-264.

[9] Verheyden G, van Duijnhoven HJ, Burnett M, Littlewood J, Kunkel D, Ashburn AM, Kinematic analysis of head, trunk, and pelvis movement when people early after stroke reach sideways. Neurorehabil Neural Repair. 2011; 25(7): 656-663.

[10] Hesse SA, Jahnke MT, Bertelt CM, Schreiner C, Lücke D, Mauritz KH. Gait outcome in ambulatory hemiparetic patients after a 4-week comprehensive rehabilitation program and prognostic factors. Stroke. 1994; 25(10): 1999-2004.

[11] Carr HJ. Stroke rehabilitation guidelines for exercise and training to optimize motor skill. New York: Elsevier Science Ltd. 2003.

[12] Wagenaar RC, Meijer OG, van Wieringen PC, Kuik DJ, Hazenberg GJ, Lindeboom J, Wichers F, Rijswijk H. The functional recovery of stroke: A comparison between neuro-developmental treatment and the brunnstrom method. Scand J Rehabil Med. 1990; 22: 1-8.

[13] Ada L, Dorsch S, Canning CG. Strengthening interventions increase strength and improve activity after stroke: A systematic review. Aust J Physiother. 2006; 52: 241-248.

[14] Dickstein R, Hocherman S, Pillar T, Shaham R. Stroke rehabilitation. Three exercise therapy approaches. Phys Ther. 1986; 66: 1233-1238.

[15] Mehrholz J, Pohl M, Elsner B. Treadmill training and body weight support for walking after stroke. Cochrane Database Syst Rev. 2014; 23.

[16] Virk S, McConville KM, Virtual reality applications in improving postural control and minimizing falls. Conf Proc IEEE Eng Med Biol Soc. 2006; 1: 2694-2697.

[17] Kim JH, Jang SH, Kim CS, Jung JH, You JH, Use of virtual reality to enhance balance and ambulation in chronic stroke: A double-blind, randomized controlled study. Am J Phys Med Rehabil. 2009; 88(9): 693-701.

[18] Mirelman A, Bonato P, Deutsch JE, Effects of training with a robot-virtual reality system compared with a robot alone on the gait of individuals after stroke. Stroke. 2009; 40(1): 169-174.

[19] Hesse S, Treadmill training with partial body weight support after stroke: A review. NeuroRehabilitation. 2008; 23(): $55-65$.

[20] McCain KJ, Pollo FE, Baum BS, Coleman SC, Baker S, Smith PS, Locomotor treadmill training with partial body-weight support before overground gait in adults with acute stroke: A pilot study. Arch Phys Med Rehabil. 2008; 89: 684-691.

[21] Visintin M., Barbeau H, Korner-Bitensky N, Mayo NE, A new approach to retrain gait in stroke patients through body weight support and treadmill stimulation. Stroke. 1998; 29(6): 1122-1128.

[22] Hesse S, Konrad M, Uhlenbrock D, Treadmill walking with partial body weight support versus floor walking in hemiparetic subjects. Arch Phys Med Rehabil. 1999; 80: 421-427.

[23] Walker ML, Ringleb SI, Maihafer GC, Walker R, Crouch JR, Van Lunen B and et al, Virtual reality-enhanced partial body weight-supported treadmill training post-stroke: Feasibility and effectiveness in 6 subjects; 91(1): 115-122.

[24] Fung J, Richards CL, Malouin F, McFadyen BJ, Lamontagne A, A treadmill and motion coupled virtual reality system for gait training post-stroke. Cyberpsychol Behav. 2006; 9(2): 157-162.

[25] McGinley JL, Baker R, Wolfe R, Morris ME. The reliability of three-dimensional kinematic gait measurements: A systematic review. Gait and Posture. 2009; 29: 360-369.

[26] Yavuzer G, Oken O, Elhan A, Stam HJ. Gait Posture. Repeatability of lower limb three-dimensional kinematics in patients with stroke. 2008; 27: 31-35.

[27] Kadaba MP, Ramakrishnan HK, Wootten ME. Measurement of lower extremity kinematics during level walking. J orthop Res. 1990; 8: 383-92.

[28] Noha DK, Lee NG, You JH. A novel spinal kinematic analysis using X-Ray imaging and vicon motion analysis: A case study. Bio-Medical Materials and Engineering. 2014; 24: 593-598.

[29] Chen G, Patten C, Treadmill training with harness support: Selection of parameters for individuals with post-stroke hemiparesis. J Rehabil Res Dev. 2006; 43(4): 485-498. 
[30] Kautz SA, Bowden MG, Clark DJ, Neptune RR, Comparison of motor control deficits during treadmill and over-ground walking post-stroke. Neurorehabil Neural Repair. 2011; 25(8): 756-765.

[31] Hesse S, Helm B, Krajnik J, Gregoric M, Mauritz KH, Treadmill training with partial body-weight support: Influence of body weight release on the gait of hemiparetic patients. J Neurol Rehabil. 1997; 11(1): 15-20.

[32] McCain KJ, Pollo FE, Baum BS, Coleman SC, Baker S, Smith PS, Locomotor treadmill training with partial bodyweight support before overground gait in adults with acute stroke: A pilot study. Arch Phys Med Rehabil. 2008; 89(4): 684-691.

[33] Kelley CP, Childress J, Boake C, Noser EA. Over-ground and robotic-assisted locomotor training in adults with chronic stroke: A blinded randomized clinical trial. Disabil Rehabil Assist Technol. 2013; 8(2): 161-168.

[34] Bonnyaud C, Pradon D, Boudarham J, Robertson J, Vuillerme N, Roche N. Effects of gait training using a robotic constraint (Lokomat ${ }^{\circledR}$ ) on gait kinematics and kinetics in chronic stroke patients. J Rehabil Med. 2014; 46: 132-138.

[35] Bonnyaud C, Zory R, Boudarham J, Pradon D, Bensmail D, Roche N. Effect of a robotic restraint gait training versus robotic conventional gait training on gait parameters in stroke patients. Exp Brain Res. 2014; 232: 31-42.

[36] Stoller O, Waser M, Stammler L, Schuster C. Evaluation of robot-assisted gait training using integrated biofeedback in neurologic disorders. Gait and Posture. 2012; 35: 595-600.

[37] Swinnen E, Baeyens JP, Knaepen K, Michielsen M, Hens G, Clijsen R, Goossens M, Buyl R, Meeusen R, Kerckhofs E. Walking with robot assistance: The influence of body weight support on the trunk and pelvis kinematics. Disabil Rehabil Assist Technol. 2014; 11.

[38] Hacmon RR, Krasovsky T, Lamontagne A, Levin MF, Deficits in intersegmental trunk coordination during walking are related to clinical balance and gait function in chronic stroke. J Neurol Phys Ther. 2012; 36(4): 173-181.

[39] Huang QH, Zheng YP, Chen X, He JF, Shi H. A system for the synchronized recording of sonomyography, electromyography and joint angle. The Open Biomedical Engineering Journal. 2007; 1: 77-84. 